\title{
CHARACTERIZATION FOR THE SOLVABILITY OF NONLINEAR PARTIAL DIFFERENTIAL EQUATIONS
}

\author{
ELEMER E. ROSINGER
}

\begin{abstract}
Within the nonlinear theory of generalized functions introduced earlier by the author a number of existence and regularity results have been obtained. One of them has been the first global version of the Cauchy-Kovalevskaia theorem, which proves the existence of generalized solutions on the whole of the domain of analyticity of arbitrary analytic nonlinear PDEs. These generalized solutions are analytic everywhere, except for closed, nowhere dense subsets which can be chosen to have zero Lebesgue measure.

This paper gives a certain extension of that result by establishing an algebraic necessary and sufficient condition for the existence of generalized solutions for arbitrary polynomial nonlinear PDEs with continuous coefficients. This algebraic characterization, given by the so-called neutrix or off diagonal condition, is proved to be equivalent to certain densely vanishing conditions, useful in the study of the solutions of general nonlinear PDEs.
\end{abstract}

\section{INTRODUCTION}

A comprehensive nonlinear theory of generalized functions aimed to solve large classes of nonlinear PDEs has been developed in [8, 9, 10, 11]. One of the results obtained, [10, pp. 259-266], [12], is a global version of the CauchyKovalevskaia theorem. This result, which is a first of its kind in the literature, proves the global existence of generalized solutions on the whole of the domain of analyticity of arbitrary analytic nonlinear PDEs, with associated noncharacteristic analytic initial data. Moreover, these global generalized solutions prove to be analytic on the whole of the domain of analyticity of the respective PDEs, except for closed, nowhere dense subsets which can be chosen to have zero Lebesgue measure. Surprisingly, the mentioned nonlinear theory of generalized functions does not need functional analytic methods and it only uses calculus in Euclidean spaces, as well as algebraic methods in rings of continuous or smooth functions on Euclidean spaces.

In this paper we consider arbitrary polynomial nonlinear PDEs with continuous coefficients

$$
\sum_{1 \leq i \leq h} c_{i}(x) \prod_{1 \leq j \leq k_{i}} D^{P_{i j}} U(x)=f(x), \quad x \in \Omega,
$$

where $\Omega \subset \mathbf{R}^{n}$ is nonvoid open, $c_{i}, f \in \mathscr{C}^{0}(\Omega)$ are given, $p_{i j} \in \mathbf{N}^{n}$, while $U: \Omega \rightarrow \mathbf{R}$ is the unknown function.

Received by the editors June 15, 1989 and, in revised form, December 12, 1989.

1991 Mathematics Subject Classification. Primary 35A10, 35D05, 35G20. 
The aim of the paper is to establish a necessary and sufficient condition for the existence of generalized solutions for PDEs in (1.1). This characterization happens to be of a simple and purely algebraic nature and it is given by a particular case of the so-called neutrix condition. In its general form the neutrix or off diagonal condition also characterizes the existence of the differential algebras of generalized functions introduced in $[8,9,10,11]$, algebras which contain the L. Schwartz distributions, as well as the generalized solutions of the PDEs in (1.1) and the global generalized solutions of analytic nonlinear PDEs mentioned earlier.

Obviously, the nonlinear PDEs in (1.1) are partly more general than the arbitrary analytic nonlinear PDEs. To that extent, the existence of generalized solutions, more precisely, their characterization, proved in this paper is an extension of the mentioned global version of the Cauchy-Kovalevskaia theorem.

The reason we consider the particular, polynomial form of nonlinear PDEs in (1.1) is that they allow for a rather simple and direct algebraic treatment leading to the mentioned neutrix characterization for the existence of generalized solutions. At the cost of certain technical complications, a similar result can be obtained for arbitrary continuous nonlinear PDEs

$$
F\left(x, U(x), \ldots, D^{p} U(x), \ldots\right)=0, \quad x \in \Omega,
$$

where $F$ is any real valued function, continuous in all its arguments. Such a result is obviously a full scale extension of the mentioned global version of the Cauchy-Kovalevskaia theorem. Details in this respect will be presented in a subsequent paper.

A particular case of the nonlinear theory of generalized functions presented in $[8,9,10,11,12]$ has been introduced and developed independently in $[2,3]$, see details in [10, pp. 49-192], [11, pp. 301-366], as well as [1]. Within this particular theory large classes of earlier unsolved or distributionally unsolvable linear and nonlinear PDEs have found generalized solutions. However, the question remains open whether existence results such as those in this paper, or the mentioned global version of the Cauchy-Kovalevskaia theorem, can be obtained within the particular framework in $[2,3]$.

\section{THE NOTION OF GENERALIZED SOLUTION}

Let us denote by $T(D)$ the polynomial nonlinear partial differential operator in the left-hand term of (1.1) and define its order by

$$
m=\max \left\{\left|p_{i j}\right| \mid 1 \leq i \leq h, 1 \leq j \leq k_{i}\right\} .
$$

For the sake of brevity it will be convenient to adapt and restrict the general framework in $[8,9,10,11]$ to the particular case of PDEs in (1.1) and correspondingly simplify some of the notation.

The spaces of generalized functions used in the sequel will be quotient vector spaces

$$
E=\mathscr{S} / \mathscr{V}
$$

or quotient algebras

$$
A=\mathscr{A} / \mathscr{I}
$$


where we have

$$
\mathscr{V} \subset \mathscr{S} \subset\left(\mathscr{C}^{m}(\Omega)\right)^{\mathbf{N}}
$$

with $\mathscr{V}$ and $\mathscr{S}$ vector subspaces, and respectively

$$
\mathscr{I} \subset A \subset\left(\mathscr{C}^{0}(\Omega)\right)^{\mathbf{N}}
$$

where $\mathscr{A}$ is a subalgebra, while $\mathscr{I}$ is an ideal in $\mathscr{A}$. Further, we shall require that the inclusion diagrams hold

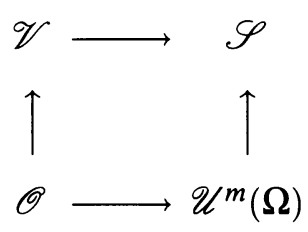

and

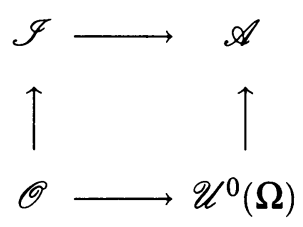

where $\mathscr{O}$ denotes null vector subspace, while $\mathscr{U}^{m}(\Omega)$ is the diagonal in $\left(\mathscr{C}^{m}(\Omega)\right)^{\mathbf{N}}$, that is

$$
\mathscr{U}^{m}(\Omega)=\left\{u(\psi) \mid \psi \in \mathscr{C}^{m}(\Omega)\right\}
$$

with $u(\psi)=(\psi, \psi, \psi, \ldots, \psi, \ldots) \in\left(\mathscr{C}^{m}(\Omega)\right)^{\mathbf{N}}$, and similarly for $\mathscr{U}^{0}(\Omega)$. Note that $\mathscr{U}^{m}(\Omega) \subset \mathscr{U}^{0}(\Omega)$ and both are subalgebras.

Finally, each of the inclusion diagrams (2.6) and (2.7) is supposed to satisfy the neutrix condition, that is

$$
\mathscr{V} \cap \mathscr{U}^{m}(\Omega)=\mathscr{O}
$$

respectively

$$
\mathscr{I} \cap \mathscr{U}^{0}(\Omega)=\mathscr{O} \text {. }
$$

It is easy to see that (2.9) and (2.10) respectively aie the necessary and sufficient conditions for the existence of the vector space and algebra embeddings

$$
\mathscr{C}^{m}(\Omega) \rightarrow E=\mathscr{S} / \mathscr{V}, \quad \psi \mapsto u(\psi)+\mathscr{V}
$$

and

$$
\mathscr{C}^{0}(\Omega) \rightarrow A=\mathscr{A} / \mathscr{I}, \quad \psi \mapsto u(\psi)+\mathscr{I} .
$$

From a geometric point of view, the neutrix condition (2.9) means that the vector subspace $\mathscr{V}$ is off diagonal in the cartesian product $\left(\mathscr{C}^{m}(\Omega)\right)^{\mathbf{N}}$. Similarly, the neutrix condition (2.10) means that the ideal $\mathscr{I}$ is off diagonal in the cartesian product $\left(\mathscr{C}^{0}(\Omega)\right)^{\mathbf{N}}$. For further comment on the neutrix condition see $\S 5$.

Assuming now that

$$
D^{p} \mathscr{V} \subset \mathscr{I}, \quad D^{p} \mathscr{S} \subset \mathscr{A}, \quad \forall p \in \mathbf{N}^{n},|p| \leq m,
$$


we can obviously extend the classical partial derivative operators

$$
D^{p}: \mathscr{C}^{m}(\Omega) \rightarrow \mathscr{C}^{0}(\Omega), \quad \forall p \in \mathbf{N}^{n},|p| \leq m
$$

to

$$
D^{p}: E \rightarrow A, \quad \forall p \in \mathbf{N}^{n},|p| \leq m
$$

by defining them according to

$$
D^{p}(s+\mathscr{V})=D^{p} s+\mathscr{I}, \quad \forall s \in \mathscr{S}, p \in \mathbf{N}^{n},|p| \leq m
$$

where $D^{p} s=\left(D^{p} s_{0}, \ldots, D^{p} s_{\nu}, \ldots\right)$ for every $s=\left(s_{0}, \ldots, s_{\nu}, \ldots\right) \in$ $\left(\mathscr{C}^{m}(\Omega)\right)^{\mathbf{N}}$. It follows that, as an extension of the classical partial differential operator

$$
T(D): \mathscr{C}^{m}(\Omega) \rightarrow \mathscr{C}^{0}(\Omega)
$$

we can define the mapping

$$
T(D): E \rightarrow A
$$

by

$$
T(D)(s+\mathscr{V})=T(D) s+\mathscr{I}, \quad \forall s \in \mathscr{S}
$$

where $T(D) s=\left(T(D) s_{0}, \ldots, T(D) s_{\nu}, \ldots\right)$ for every $s=\left(s_{0}, \ldots, s_{\nu}, \ldots\right) \in$ $\left(\mathscr{C}^{m}(\Omega)\right)^{\mathbf{N}}$. Indeed, it is easy to see that the definition in $(2.18),(2.19)$ is correct. For that, we note the following. Let $s, v \in\left(\mathscr{C}^{m}(\Omega)\right)^{\mathbf{N}}$, then in view of $(1.1)$, we obtain

$$
T(D)(s+v)=T(D) s+\sum_{\alpha} s_{\alpha} \cdot D^{p_{\alpha}} v
$$

where $s_{\alpha}$ are products of $c_{i}, D^{p_{i j}} s$ and possibly $D^{p_{i j}} v$, while $p_{\alpha}$ are some of the $p_{i j}$. In this way, if $s \in \mathscr{S}$ and $v \in \mathscr{V}$, then the inclusions (2.13) imply that

$$
\sum_{\alpha} s_{\alpha} \cdot D^{p_{\alpha}} v \in \mathscr{I}
$$

therefore

$$
T(D)(s+v)-T(D) s \in \mathscr{J}
$$

hence (2.19) is a valid definition.

Now, as an extension of the notion of classical solution, we can define the generalized solutions for the nonlinear PDE in (1.1) as being given by all generalized functions

$$
U=s+\mathscr{V} \in E=\mathscr{S} / \mathscr{V}
$$

such that, in the sense of $(2.18)$, we have

$$
T(D) U=f
$$

where we note that, in view of (2.12), we have $f \in A$.

As shown in $[8,9,10,11,12]$, this notion of generalized solution is an extension of the notion of distributional or weak solution, see also $[2,3,1,5,6$, 
7]. In particular, one can construct the spaces of generalized functions in (2.2) and (2.3) in such a way that they contain the distributions, that is

$$
\mathscr{D}^{\prime}(\Omega) \subset E, \quad \mathscr{D}^{\prime}(\Omega) \subset A .
$$

Moreover, in (2.18), and therefore in (2.23), $E$ itself can be an algebra. In fact, one can have $E=A$, in which case they are differential algebras and contain the distributions.

However, for the purposes of this paper, it is convenient to allow for the generality of the framework in (2.18) and (2.23).

We note that within this framework, the only connection needed between $E$ and $A$ is that in (2.13), which for convenience we shall denote by

$$
E \stackrel{m}{\leq} A .
$$

In rest, $E$ can be arbitrary within the conditions (2.2), (2.4), (2.6) and (2.9). We shall denote by

$$
V S^{m}(\Omega)
$$

the set of all such quotient vector spaces $E$ of generalized functions. Similarly, $A$ can be arbitrary, provided that it satisfies the conditions (2.3), (2.5), (2.7) and (2.10). And we denote by

$$
A L(\Omega)
$$

the set of all these quotient algebras $A$ of generalized functions.

\section{ThE PROBLEM OF SOLVABILITY OF NONLINEAR PDES}

Given the polynomial nonlinear PDE with continuous coefficients in (1.1),

$$
T(D) U(x)=f(x), \quad x \in \Omega,
$$

the problem of its solvability will be formulated as follows.

For sequences of smooth functions

$$
s \in\left(\mathscr{C}^{m}(\Omega)\right)^{\mathbf{N}}
$$

we shall find the necessary and sufficient condition for the existence of spaces of generalized functions $E=\mathscr{S} / \mathscr{V} \in V S^{m}(\Omega)$ and $A=\mathscr{A} / \mathscr{I} \in A L(\Omega)$ with

$$
E \stackrel{m}{\leq} A
$$

and such that the generalized function

$$
U=s+\mathscr{V} \in E=\mathscr{S} / \mathscr{V}
$$

satisfies the nonlinear PDE

$$
T(D) U=f
$$

in the sense of (2.18). For convenience, we shall denote the nonlinear PDE in (3.5) by $\mathscr{E}$.

\section{NeUtriX CHARACTERIZATION FOR THE SOLVABILITY OF NONLINEAR PDES}

Let us now make more explicit the above solvability problem in (3.2)-(3.5). Condition (3.3) is nothing but (2.13), while condition (3.4) is equivalent to

$$
s \in \mathscr{S} \text {. }
$$


Finally, in view of (2.19), condition (3.5) is equivalent to

$$
w_{s}=T(D) s-u(f) \in \mathscr{I} .
$$

It follows that the solvability problem in (3.2)-(3.5) is equivalent to finding $E=\mathscr{S} / \mathscr{V} \in V S^{m}(\Omega)$ and $A=\mathscr{A} / \mathscr{I} \in A L(\Omega)$ such that (2.13), (4.1) and (4.2) are satisfied.

In view of the fact that condition (4.2) only involves the equation $\mathscr{E}$, the sequence $s$ and the ideal $\mathscr{I}$, without relating to $E=\mathscr{S} / \mathscr{V}$ or $\mathscr{A}$, we shall deal with it first. For that purpose, given a sequence of continuous functions $w \in\left(\mathscr{C}^{0}(\Omega)\right)^{\mathbf{N}}$, let us define the quotient algebra

$$
A_{w}=\mathscr{A}_{w} / \mathscr{I}_{w}
$$

where $\mathscr{A}_{w}$ is the subalgebra in $\left(\mathscr{C}^{0}(\Omega)\right)^{\mathbf{N}}$ generated by $\{w\} \cup \mathscr{U}^{0}(\Omega)$, while $\mathscr{I}_{w}$ is the ideal in $\mathscr{A}_{w}$ generated by $w$, thus

$$
\mathscr{I}_{w}=w \cdot \mathscr{A}_{w} \text {. }
$$

It follows easily that

$$
A_{w}=\mathscr{A}_{w} / \mathscr{I}_{w} \in A L(\Omega) \Leftrightarrow \mathscr{I}_{w} \cap \mathscr{U}^{0}(\Omega)=\mathscr{O} .
$$

The interest in the quotient algebra $A_{w}$ comes from the following characterization.

Proposition 1. If $w \in\left(\mathscr{C}^{0}(\Omega)\right)^{\mathbf{N}}$ then the three conditions below are equivalent

$$
\begin{gathered}
\exists A=\mathscr{A} / \mathscr{I} \in A L(\Omega): w \in \mathscr{I} . \\
A_{w}=\mathscr{A}_{w} / \mathscr{J}_{w} \in A L(\Omega) . \\
\mathscr{I}_{w} \cap \mathscr{U}^{0}(\Omega)=\mathscr{O} .
\end{gathered}
$$

Further, if (4.6) holds, then we have the inclusion diagram

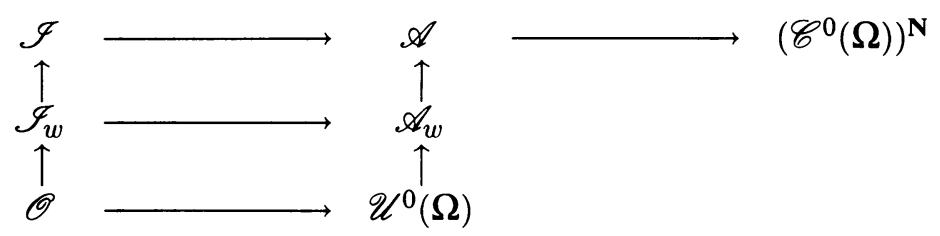

Proof. In view of (4.5) the conditions (4.7) and (4.8) are equivalent.

Let us assume (4.6). Then (2.7) yields $\{w\} \cup \mathscr{U}^{0}(\Omega) \subset \mathscr{I} \cup \mathscr{A} \subset \mathscr{A}$ therefore $\mathscr{A}_{w} \subset \mathscr{A}$. Further, in view of (4.4) we obviously have

$$
w \in \mathscr{I} \Rightarrow w \cdot \mathscr{A} \subset \mathscr{I} \Rightarrow w \cdot \mathscr{A}_{w} \subset \mathscr{I} \Rightarrow \mathscr{I}_{w} \subset \mathscr{I} .
$$

Thus in view of $(2.10)$ we obtain

$$
\mathscr{I}_{w} \cap \mathscr{U}^{0}(\Omega) \subset \mathscr{I} \cap \mathscr{U}^{0}(\Omega)=\mathscr{O}
$$

and then (4.5) ends the proof of (4.8). Meanwhile we note that (4.9) has been proved as well.

Conversely, if (4.8) holds, then we can take $A=A_{w}$ in (4.6).

Returning to condition (4.2), it is now obvious that it can be written in the equivalent neutrix or off diagonal form

$$
\mathscr{I}_{w_{s}} \cap \mathscr{U}^{0}(\Omega)=\mathscr{O}
$$


and in view of (4.9), $\mathscr{J}_{w_{s}}$ is the smallest ideal for which (4.10) has to hold.

In order to further explicitate the condition (4.10) it is useful to impose the following rather natural and mild restriction on the polynomial nonlinear partial differential operators $T(D)$ in $\mathscr{E}$.

We call $T(D)$ nontrivial on $\Omega$, if and only if, when restricted to any $\mathscr{C}\left(\Omega^{\prime}\right)$, with $\Omega^{\prime} \subset \Omega$ nonvoid and open, the range of the mapping $T(D)$ in (2.17) is an infinite subset of $\mathscr{C}^{0}\left(\Omega^{\prime}\right)$.

It is equally convenient to impose a similarly natural and mild restriction on the sequences $s$ in (3.2), which through (3.4) are supposed to give the generalized solutions for $\mathscr{E}$. For that, we shall replace (3.2) by the condition

$$
s \in\left(\mathscr{C}^{m}(\Omega)\right)_{\mathscr{E}}^{\mathbf{N}}
$$

Here $\left(\mathscr{C}^{m}(\Omega)\right)_{\mathscr{C}}^{\mathbf{N}}$ is the subset of all the sequences $s \in\left(\mathscr{C}^{m}(\Omega)\right)^{\mathbf{N}}$ which do not satisfy the condition

$\exists \Omega^{\prime} \subset \Omega$ nonvoid, open:

$$
\begin{aligned}
& \exists t \text { subsequence in } s, g \in \mathscr{C}^{0}\left(\Omega^{\prime}\right), \quad g \neq f: \\
& \forall \nu \in \mathbf{N}: \\
& \quad T(D) t_{\nu}=g, \quad \text { on } \Omega^{\prime} .
\end{aligned}
$$

Proposition 2. If $T(D)$ is nontrivial on $\Omega$, then

$$
\left(\mathscr{C}^{m}(\Omega)\right)_{\mathscr{E}}^{\mathbf{N}} \neq \varnothing .
$$

Proof. Assume that $\left(\mathscr{C}^{m}(\Omega)\right)_{\mathscr{E}}^{\mathbf{N}}=\varnothing$ then (4.12) implies that

$$
\begin{aligned}
& \forall s \in\left(\mathscr{C}^{m}(\Omega)\right)^{\mathbf{N}}: \\
& \exists \Omega^{\prime} \subset \Omega \text { nonvoid, open: } \\
& \exists t \text { subsequence in } s, g \in \mathscr{C}^{0}\left(\Omega^{\prime}\right), \quad g \neq f: \\
& \exists \nu \in \mathbf{N}: \\
& \quad T(D) t_{\nu}=g, \quad \text { on } \Omega^{\prime} .
\end{aligned}
$$

But (4.14) obviously implies that the range of the mapping $T(D)$ in (2.17), when restricted to $\mathscr{C}^{m}\left(\Omega^{\prime}\right)$, is a finite subset of $\mathscr{C}^{0}\left(\Omega^{\prime}\right)$.

At this stage we are led to introduce, see [9, p. 39]

$$
\mathscr{R}(\Omega)
$$

as the set of all the sequences $w \in\left(\mathscr{C}^{0}(\Omega)\right)^{\mathbf{N}}$ for which the following condition does not hold

$$
\exists \Omega^{\prime} \subset \Omega \text { nonvoid, open: }
$$

$\exists z$ subsequence in $w, h \in \mathscr{C}^{0}\left(\Omega^{\prime}\right), \quad h \neq 0$ :

$\forall \nu \in \mathbf{N}$ :

$$
z_{\nu}=h, \quad \text { on } \Omega^{\prime}
$$

Indeed, we obviously have the property

$$
\begin{aligned}
\forall s & \in\left(\mathscr{C}^{m}(\Omega)\right)^{\mathbf{N}}: \\
s & \in\left(\mathscr{C}^{m}(\Omega)\right)_{\mathscr{C}}^{\mathbf{N}} \Leftrightarrow w_{s} \in \mathscr{R}(\Omega) .
\end{aligned}
$$

However, the important property of the set of sequences $\mathscr{R}(\Omega)$ related to condition (4.10) is the following. 
Proposition 3. If $w \in \mathscr{R}(\Omega)$ then

$$
\mathscr{I}_{w} \cap \mathscr{U}^{0}(\Omega)=\mathscr{O} \text {. }
$$

Proof. It is easy to see that an element of the intersection in (4.18) has the form

$$
u(\psi)=w \cdot u\left(\psi_{0}\right)+\cdots+w^{l+1} \cdot u\left(\psi_{l}\right)
$$

where $l \in \mathbf{N}$ and $\psi, \psi_{0}, \ldots, \psi_{l} \in \mathscr{C}^{0}(\Omega)$. Therefore in order to prove (4.18), it suffices to show that $\psi=0$ on $\Omega$. Assume that this is not the case and $\Omega^{\prime} \subset \Omega$ is nonvoid, open, such that

$$
\psi(x) \neq 0, \quad \forall x \in \Omega^{\prime} .
$$

Denoting by $w_{\nu}$, with $\nu \in \mathbf{N}$, the continuous functions on $\Omega$ that are the terms in the sequence $w$, the relation (4.19) written term by term, yields

$$
\begin{aligned}
\left(w_{\nu}(x)\right)^{l+1} \cdot \psi_{l}(x)+\cdots+w_{\nu}(x) \cdot \psi_{0}(x)+(-\psi(x)) & =0, \\
\forall \nu & \in \mathbf{N}, \quad x \in \Omega .
\end{aligned}
$$

Therefore (4.20) will imply that the infinite matrix

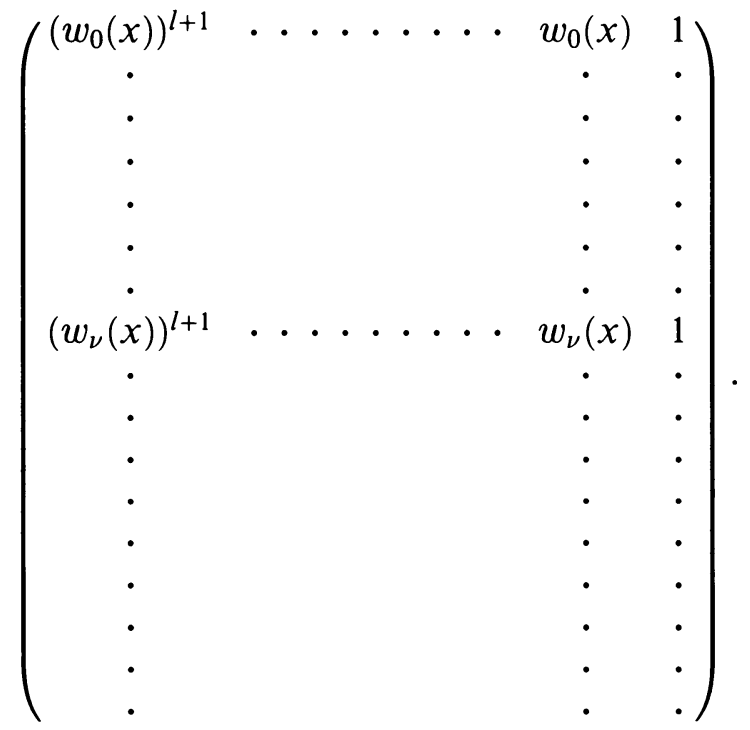

has rank at most $l+1$, for any given $x \in \Omega^{\prime}$.

Now, a well-known property of Vandermonde determinants implies that the infinite sequence of numbers

$$
w_{0}(x), \ldots, w_{\nu}(x), \ldots,
$$

contains at most $l+1$ different terms, for any given $x \in \Omega^{\prime}$. Therefore Lemma 1 below will grant the existence of a closed, nowhere dense subset $\Gamma^{\prime} \subset \Omega^{\prime}$, such that each $x \in \Omega^{\prime} \backslash \Gamma^{\prime}$ possesses an open neighborhod $\Omega^{\prime \prime} \subset \Omega^{\prime} \backslash \Gamma^{\prime}$, with the property that the infinite sequence of functions $w_{0}, \ldots, w_{\nu}, \ldots$ when restricted to $\Omega^{\prime \prime}$, contains only a finite number of different functions. In other words, there exists a subsequence $w^{\prime \prime}$ in $w$ and $\psi^{\prime \prime} \in \mathscr{C}^{0}\left(\Omega^{\prime \prime}\right)$ such that

$$
w^{\prime \prime}=u\left(\psi^{\prime \prime}\right) \text { on } \Omega^{\prime \prime} \text {. }
$$

Now, $w \in \mathscr{R}(\Omega)$ will imply that $\psi^{\prime \prime}=0$ on $\Omega^{\prime \prime}$. And then (4.22) together with (4.21) will contradict (4.20). 
Lemma 1. Suppose the sequence $w=\left(w_{0}, \ldots, w_{\nu}, \ldots\right)$ of continuous functions on $\Omega$ is such that for any given $x \in \Omega$, the sequence of numbers

$$
w_{0}(x), \ldots, w_{\nu}(x), \ldots
$$

contains only a finite number of different terms. Then there exists a closed, nowhere dense subset $\Gamma \subset \Omega$, such that the sequence of functions $w_{0}, \ldots, w_{\nu}, \ldots$ restricted to a suitable neighborhood of any given $x \in \Omega \backslash \Gamma$, contains only a finite number of different terms.

Proof. Denote by $\Gamma$ the set of all points $x \in \Omega$ such that the sequence of functions $w_{0}, \ldots, w_{\nu}, \ldots$ when restricted to any neighborhood of $x$, contains infinitely many different terms. It is easy to see that $\Gamma$ is closed. Therefore it only remains to prove that $\Gamma$ has no interior. It suffices to show that

$$
\Gamma \neq \Omega \text {. }
$$

Indeed, denote $\Omega^{\prime}=\operatorname{int} \Gamma$ and assume $\Omega^{\prime} \neq \varnothing$. Then $\Gamma^{\prime}$ corresponding as above to $\Omega^{\prime}$, will satisfy $\Gamma^{\prime}=\Omega^{\prime}$, hence contradicting (4.23).

In order to obtain (4.23), the Baire category argument will be used in two successive steps.

First, for $\mu \in \mathbf{N}$, define the closed set

$$
\Delta_{\mu}=\left\{x \in \Omega \mid \forall \nu \in \mathbf{N}, \nu \geq \mu+1: \exists \lambda \in \mathbf{N}, \lambda \leq \mu: w_{\nu}(x)=w_{\lambda}(x)\right\},
$$

then obviously

$$
\Omega=\bigcup_{\mu \in \mathbf{N}} \Delta_{\mu}
$$

therefore the Baire category argument implies that int $\Delta_{\mu} \neq \varnothing$ for a certain $\mu \in \mathbf{N}$. Denote $\Omega^{\prime}=\operatorname{int} \Delta_{\mu}$. We shall prove that

$$
\Omega^{\prime} \cap(\Omega \backslash \Gamma) \neq \varnothing .
$$

Denote for $\rho \in \mathbf{N}$,

$$
\begin{aligned}
\Delta_{\rho}^{\prime}=\left\{x \in \Omega^{\prime} \mid \forall \lambda, \nu \in \mathbf{N}, \quad \lambda<\nu \leq \mu:\right. \\
\left.w_{\lambda}(x) \neq w_{\nu}(x) \Rightarrow\left|w_{\lambda}(x)-w_{\nu}(x)\right| \geq 1 /(\rho+1)\right\}
\end{aligned}
$$

then we have

$$
\Omega^{\prime}=\bigcup_{\rho \in \mathbf{N}} \Delta_{\rho}^{\prime}
$$

Indeed, denote for $x \in \Omega^{\prime}$

$$
M_{x}=\left\{(\lambda, \nu) \in \mathbf{N} \times \mathbf{N} \mid \lambda<\nu \leq \mu, w_{\lambda}(x) \neq w_{\nu}(x)\right\}
$$

and take $\rho \in \mathbf{N}$, such that

$$
1 /(\rho+1) \leq \min \left\{\left|w_{\lambda}(x)-w_{\nu}(x)\right| \mid(\lambda, \nu) \in M_{x}\right\}
$$

then obviously $x \in \Delta_{\rho}^{\prime}$.

Now we show that

$$
\Delta_{\rho}^{\prime} \text { closed, } \quad \forall \rho \in \mathbf{N} \text {. }
$$

Indeed, denoting

$$
M=\{(\lambda, \nu) \in \mathbf{N} \times \mathbf{N} \mid \lambda<\nu \leq \mu\}
$$


we have

$$
\begin{aligned}
\Delta_{\rho}^{\prime}=\bigcup_{K \subset M}\left(\bigcup_{(\lambda, \nu) \in K}\left\{x \in \Omega^{\prime}|| w_{\lambda}(x)-w_{\nu}(x) \mid \geq 1 /(\rho+1)\right\}\right. \\
\left.\cap \bigcup_{(\lambda, \nu) \in M \backslash K}\left\{x \in \Omega^{\prime} \mid w_{\lambda}(x)=w_{\nu}(x)\right\}\right) .
\end{aligned}
$$

But (4.25) and (4.26) together with the Baire category argument imply that int $\Delta_{\rho}^{\prime} \neq \varnothing$ for a certain $\rho \in \mathbf{N}$. Denote then $\Omega^{\prime \prime}=$ int $\Delta_{\rho}^{\prime}$. The proof of $(4.24)$ will obviously be complete if we show that

$$
\Omega^{\prime \prime} \subset \Omega \backslash \Gamma \text {. }
$$

Assume therefore $x \in \Omega^{\prime \prime}$ and $V \subset \Omega^{\prime \prime}$ an open, connected neighbourhood of $x$. We shall prove that the sequence of functions $w_{0}, \ldots, w_{\nu}, \ldots$ when restricted to $V$, contains at most $\mu+1$ different terms. Indeed, if $\nu \in \mathbf{N}$, $\nu \geq \mu+1$, then $w_{\nu}(x)=w_{\lambda}(x)$, for a certain $\lambda \in \mathbf{N}, \lambda \leq \mu$, since $x \in V \subset$ $\Omega^{\prime \prime} \subset \Delta_{\rho}^{\prime} \subset \Omega^{\prime} \subset \Delta_{\mu}$. But then

$$
w_{\nu}=w_{\lambda} \text { on } V .
$$

Assume indeed that (4.28) is false. Then $w_{\nu}(y) \neq w_{\lambda}(y)$, for a certain $y \in V$. Denote

$$
V^{\prime}=\left\{x^{\prime} \in V \mid w_{\nu}\left(x^{\prime}\right)=w_{\lambda}\left(x^{\prime}\right)\right\}, \quad V^{\prime \prime}=\left\{x^{\prime \prime} \in V \mid w_{\nu}\left(x^{\prime \prime}\right) \neq w_{\lambda}\left(x^{\prime \prime}\right)\right\}
$$

then $x \in V^{\prime}, y \in V^{\prime \prime}, V=V^{\prime} \cup V^{\prime \prime}, V^{\prime} \cap V^{\prime \prime}=\varnothing$ and $V^{\prime}$ is obviously closed. But $V^{\prime \prime}$ is also closed, since

$$
V^{\prime \prime}=\left\{x^{\prime \prime} \in V|| w_{\nu}\left(x^{\prime \prime}\right)-w_{\lambda}\left(x^{\prime \prime}\right) \mid \geq 1 /(\rho+1)\right\}
$$

the inclusion $\supset$ being obvious, while the converse results as follows. Take $x^{\prime \prime} \in V^{\prime \prime}$, then there exists $\sigma \in \mathbf{N}, \sigma \leq \mu$, such that $w_{\nu}\left(x^{\prime \prime}\right)=w_{\sigma}\left(x^{\prime \prime}\right)$, since $\nu \geq \mu+1$ and

$$
x^{\prime \prime} \in V^{\prime \prime} \subset V \subset \Omega^{\prime \prime} \subset \Delta_{\rho}^{\prime} \subset \Omega^{\prime} \subset \Delta_{\mu} .
$$

Hence $w_{\sigma}\left(x^{\prime \prime}\right) \neq w_{\lambda}\left(x^{\prime \prime}\right)$, therefore $\sigma, \lambda \leq \mu$ and $x^{\prime \prime} \in V^{\prime \prime} \subset V \subset \Omega^{\prime \prime} \subset \Delta_{\rho}^{\prime}$ will imply that

$$
\left|w_{\nu}\left(x^{\prime \prime}\right)-w_{\lambda}\left(x^{\prime \prime}\right)\right|=\left|w_{\sigma}\left(w^{\prime \prime}\right)-w_{\lambda}\left(x^{\prime \prime}\right)\right| \geq 1 /(\rho+1)
$$

and this completes the proof of (4.29).

As the decomposition $V=V^{\prime} \cup V^{\prime \prime}$ that has been obtained contradicts the connectedness of $V$, it follows that (4.28) holds.

Now, (4.28) implies (4.27), which completes the proof of (4.24). Thus finally (4.23) has been proved.

For the sake of completeness, we note the following obvious property of the set $\mathscr{R}(\Omega)$ of sequences of continuous functions on $\Omega$,

(4.30) $\forall w \in\left(\mathscr{C}^{0}(\Omega)\right)^{\mathbf{N}}$ :

$w \in \mathscr{R}(\Omega) \Leftrightarrow\left(\forall \Omega^{\prime} \subset \Omega\right.$ nonvoid open, $z$ subsequence in $w$ :

$$
\left.\left.z\right|_{\Omega^{\prime}} \in \mathscr{R}\left(\Omega^{\prime}\right)\right)
$$

where, for $z=\left(z_{0}, \ldots, z_{\nu}, \ldots\right) \in\left(\mathscr{C}^{0}(\Omega)\right)^{\mathbf{N}}$, we denote

$$
\left.z\right|_{\Omega^{\prime}}=\left(\left.z_{0}\right|_{\Omega^{\prime}}, \ldots,\left.z_{\nu}\right|_{\Omega^{\prime}}, \ldots\right)
$$


with

$$
\left.\mathscr{C}^{0}(\Omega) \ni k \mapsto k\right|_{\Omega^{\prime}} \in \mathscr{C}^{0}\left(\Omega^{\prime}\right)
$$

being the usual restriction of the function $k$ to $\Omega^{\prime}$. Further, for any subset $\mathscr{I} \subset\left(\mathscr{C}^{0}(\Omega)\right)^{\mathbf{N}}$ let us denote

$$
\left.\mathscr{I}\right|_{\Omega^{\prime}}=\left\{\left.z\right|_{\Omega^{\prime}} \mid z \in \mathscr{I}\right\}
$$

Based on (4.30), we obtain

Corollary 1. If $w \in\left(\mathscr{C}^{0}(\Omega)\right)^{\mathbf{N}}$ then

(4.31) $w \in \mathscr{R}(\Omega) \Leftrightarrow\left(\forall \Omega^{\prime} \subset \Omega\right.$ nonvoid open, $z$ subsequence in $w$ :

$$
\left.\left.\mathscr{J}_{z}\right|_{\Omega^{\prime}} \cap \mathscr{U}^{0}\left(\mathbf{\Omega}^{\prime}\right)=\mathscr{O}\right) \text {. }
$$

Proof. The implication " $\Rightarrow$ " follows easily from (4.18) and (4.30).

Conversely, assume that $w \notin \mathscr{R}(\Omega)$. Then in view of (4.16) we obtain a subsequence $z$ in $w$, such that for a certain nonvoid and open $\Omega^{\prime} \subset \Omega$ and $h \in\left(\mathscr{C}^{0}\left(\Omega^{\prime}\right), h \neq 0\right.$, we have

$$
\left.u(h) \in \mathscr{I}_{z}\right|_{\Omega^{\prime}} \cap \mathscr{U}^{0}\left(\Omega^{\prime}\right)
$$

which contradicts the hypothesis.

Connected with the neutrix condition (4.10) we can now obtain the following result.

Corollary 2. If $s \in\left(\mathscr{C}^{m}(\Omega)\right)_{\mathscr{E}}^{\mathbf{N}}$ then, for every $\Omega^{\prime} \subset \Omega$ nonvoid and open, we have

$$
\left.\mathscr{I}_{w_{s}}\right|_{\Omega^{\prime}} \cap \mathscr{U}^{0}\left(\Omega^{\prime}\right)=\mathscr{O} \text {. }
$$

Proof. It follows from (4.17), (4.18) and (4.31).

We note that in view of (4.17), we have the following correspondent of property (4.30)

(4.33) $\forall s \in\left(\mathscr{C}^{m}(\Omega)\right)^{\mathbf{N}}$ :

$s \in\left(\mathscr{C}^{m}(\Omega)\right)_{\mathscr{E}}^{\mathbf{N}} \Leftrightarrow\left(\forall \Omega^{\prime} \subset \Omega\right.$ nonvoid open, $t$ subsequences in $s$ :

$$
\left.\left.t\right|_{\Omega^{\prime}} \in\left(\mathscr{C}^{m}\left(\Omega^{\prime}\right)\right)_{\mathscr{E}}^{\mathbf{N}}\right)
$$

which leads to

Corollary 3. If $s \in\left(\mathscr{C}^{m}(\Omega)\right)^{\mathbf{N}}$ then

(4.34) $s \in\left(\mathscr{C}^{m}(\Omega)\right)_{\mathscr{E}}^{\mathbf{N}} \Leftrightarrow\left(\forall \Omega^{\prime} \subset \Omega\right.$ nonvoid open, $t$ subsequence in $s:$

$$
\left.\left.\mathscr{I}_{w_{t}}\right|_{\Omega^{\prime}} \cap \mathscr{U}^{0}\left(\Omega^{\prime}\right)=\mathscr{O}\right) \text {. }
$$

Proof. The implication " $\Rightarrow$ " follows from (4.32) and (4.33). Conversely, (4.31) implies that $w_{s} \in \mathscr{R}(\Omega)$ thus (4.17) completes the proof.

We can summarize the above as follows.

Proposition 4. If $T(D)$ is nontrivial on $\Omega$, then $\left(\mathscr{C}^{m}(\Omega)\right)_{\mathscr{E}}^{\mathbf{N}} \neq \varnothing$ and

$$
\forall s \in\left(\mathscr{C}^{m}(\Omega)\right)_{\mathscr{E}}^{\mathbf{N}}: A_{w_{s}}=\mathscr{A}_{w_{s}} / \mathscr{I}_{w_{s}} \in A L(\Omega) .
$$

Proof. It follows from Proposition 2 and Corollary 2. 
At this point we can turn to the conditions (3.3) and (4.1). Obviously, it is easy to find $E=\mathscr{S} / \mathscr{V} \in V S^{m}(\Omega)$ such that (4.1) is satisfied. Therefore, we concentrate on satisfying condition (3.3), that is (2.13).

For that, it is useful to note that the existence of a vector space $E$ in (2.2) and of an algebra $A$ in (2.3) satisfying the conditions (2.13), (4.1) and (4.2) is equivalent to the existence of an algebra $A$ in (2.3), satisfying the following two conditions

$$
\begin{gathered}
A=\mathscr{A} / \mathscr{I} \in A L(\Omega) . \\
w_{s} \in \mathscr{I}, \quad\left\{D^{p} s\left|p \in \mathbf{N}^{n},\right| p \mid \leq m\right\} \subset \mathscr{A} .
\end{gathered}
$$

In this case we can define $E=\mathscr{S} / \mathscr{V}$ by

$$
\mathscr{S}=\left\{t \in\left(\mathscr{C}^{m}(\Omega)\right)^{\mathbf{N}}\left|\forall p \in \mathbf{N}^{n},\right| p \mid \leq m: D^{p} t \in \mathscr{A}\right\}
$$

and

$$
\mathscr{V}=\left\{v \in\left(\mathscr{C}^{m}(\Omega)\right)^{\mathbf{N}}\left|\forall p \in \mathbf{N}^{n},\right| p \mid \leq m: D^{p} v \in \mathscr{I}\right\}
$$

Indeed, it is easy to see that

$$
E=\mathscr{S} / \mathscr{V} \in V S^{m}(\Omega) \Leftrightarrow \mathscr{V} \cap \mathscr{U}^{m}(\Omega)=\mathscr{O} .
$$

Therefore, we prove now that the neutrix condition in the right-hand term of (4.40) does indeed hold. For that, assume the relation $v=u(\psi)$, for a certain $v \in \mathscr{V}$ and $\psi \in \mathscr{C}^{m}(\Omega)$. Then, for $p=(0, \ldots, 0) \in \mathbf{N}^{n}$, the relation (4.39) yields $v \in \mathscr{I}$. Thus $u(\psi)=v \in \mathscr{I} \cap \mathscr{U}^{0}(\Omega)$ since $\psi \in \mathscr{C}^{m}(\Omega) \subset \mathscr{C}^{0}(\Omega)$. Now, in view of (4.36) and hence (2.10), we obtain $v \in \mathscr{O}$.

Finally, we are near to the solution of the problem (3.2)-(3.5). Indeed, in view of Proposition 1, the conditions (4.36) and (4.37) are equivalent to the existence of the inclusion diagram

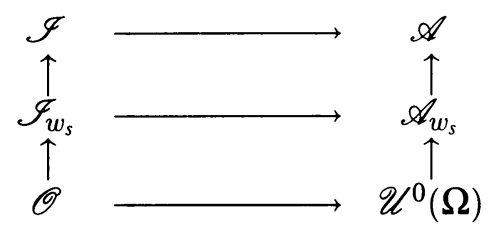

$$
\left(\mathscr{C}^{0}(\Omega)\right)^{\mathbf{N}}
$$

satisfying the additional two conditions

$$
\left\{D^{p} s\left|p \in \mathbf{N}^{n},\right| p \mid \leq m\right\} \subset \mathscr{A}
$$

and

$$
\mathscr{I} \cap \mathscr{U}^{0}(\Omega)=\mathscr{O} \text {. }
$$

We show now that in case (4.41)-(4.43) hold, one can find the smallest subalgebra $\mathscr{A}$ and ideal $\mathscr{I}$ in $\mathscr{A}$ which satisfy these conditions. Indeed, let us denote by

$$
\mathscr{A}_{S}
$$

the subalgebra in $\left(\mathscr{C}^{0}(\Omega)\right)^{\mathbf{N}}$ generated by

$$
\left\{D^{p} s\left|p \in \mathbf{N}^{n},\right| p \mid \leq m\right\} \cup \mathscr{U}^{0}(\Omega) .
$$

Then (4.42) and (2.7) imply that

$$
\mathscr{A}_{S} \subset \mathscr{A}
$$


Let us now denote by

$$
\mathscr{I}_{s}
$$

the ideal in $\mathscr{A}_{s}$ generated by $\mathscr{I}_{w_{s}}$. Then obviously $\mathscr{I}_{s}$ is the vector subspace generated by

$$
\mathscr{I}_{w_{s}} \cdot \mathscr{A}_{s}
$$

therefore (4.41) results in the inclusion

$$
\mathscr{I}_{s} \subset \mathscr{J} \text {. }
$$

In this way, whenever (4.41) holds, it can always be augmented to become the following inclusion diagram

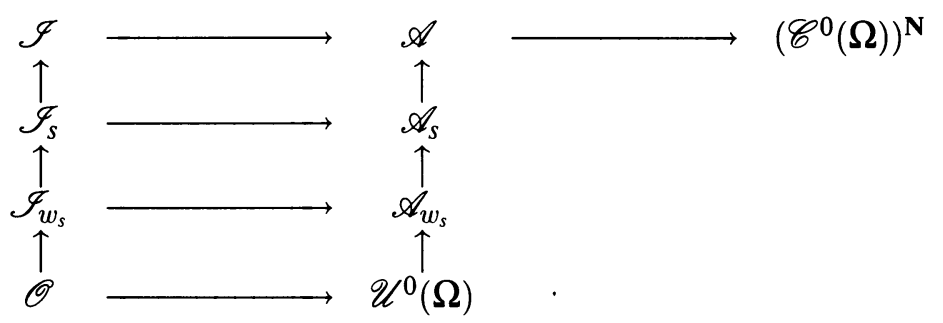

which will automatically satisfy (4.42), as a consequence of (4.45). In conclusion, the existence of inclusion diagrams (4.41) which satisfy conditions (4.42) and (4.43) is equivalent to the neutrix condition

$$
\mathscr{I}_{s} \cap \mathscr{U}^{0}(\Omega)=\mathscr{O} \text {. }
$$

Now we can summarize the results in this section and obtain the following neutrix characterization for the existence of generalized solutions.

Theorem 1. If $T(D)$ is nontrivial on $\Omega$, then

$$
\left(\mathscr{C}^{m}(\Omega)\right)_{\mathscr{E}}^{\mathbf{N}} \neq \varnothing .
$$

Given any sequence of functions

$$
s \in\left(\mathscr{C}^{m}(\Omega)\right)_{\mathscr{E}}^{\mathbf{N}}
$$

there exist $E=\mathscr{S} / \mathscr{V} \in V S^{m}(\Omega)$ and $A=\mathscr{A} / \mathscr{I} \in A L(\Omega)$ such that

$$
\begin{gathered}
E \stackrel{m}{\leq} A, \\
U=s+\mathscr{V} \in E=\mathscr{S} / \mathscr{V},
\end{gathered}
$$

and

$$
T(D) U=f \quad(\text { see }(2.18))
$$

if and only if the neutrix condition is satisfied (see (4.47))

$$
\mathscr{Y}_{s} \cap \mathscr{U}^{0}(\Omega)=\mathscr{O} \text {. }
$$

Remark 1. Let us recapitulate the main steps leading to the above algebraic characterization in (4.57) for the existence of generalized solutions.

Given on $\Omega$ a nontrivial polynomial nonlinear PDE $\mathscr{E}$ of order $m$ and with continuous coefficients

$$
T(D) U(x)=f(x), \quad x \in \Omega,
$$


and a sequence of functions

$$
s \in\left(\mathscr{C}^{m}(\Omega)\right)_{\mathscr{E}}^{\mathbf{N}}
$$

one constructs $\mathscr{A}_{S}$ in (4.44) and $\mathscr{I}_{S}$ in (4.47). Then, the neutrix condition

$$
\mathscr{I}_{s} \cap \mathscr{U}^{0}(\Omega)=\mathscr{O}
$$

is necessary and sufficient for having

$$
A_{s}=A_{s} / \mathscr{I}_{s} \in A L(\Omega)
$$

in which case one can take

$$
A=A_{s}
$$

Further, one can take

$$
E=E_{s}
$$

where

$$
E_{s}=\mathscr{S}_{s} / \mathscr{V}_{s} \in V S^{m}(\Omega)
$$

with

$$
\mathscr{S}_{s}=\left\{t \in\left(\mathscr{C}^{m}(\Omega)\right)^{\mathbf{N}}\left|\forall p \in \mathbf{N}^{n},\right| p \mid \leq m: D^{p} t \in \mathscr{A}_{s}\right\}
$$

and

$$
\mathscr{V}_{s}=\left\{v \in\left(\mathscr{C}^{m}(\Omega)\right)^{\mathbf{N}}\left|\forall p \in \mathbf{N}^{n},\right| p \mid \leq m: D^{p} v \in \mathscr{I}_{s}\right\}
$$

In this way one obtains

$$
E \stackrel{m}{\leq} A
$$

and for

$$
U=s+\mathscr{S} \in E=\mathscr{S} / \mathscr{V}
$$

the equation $\mathscr{E}$

$$
T(D) U=f
$$

is satisfied.

The above construction, which summarizes the proof of Theorem 1, gives in fact a particular pair of spaces of generalized functions $E=\mathscr{S} / \mathscr{V}$ and $A=\mathscr{A} / \mathscr{I}$. The existence of other pairs which satisfy the conditions (4.54)(4.56) can be obtained from a detailed study of the stability, generality and exactness of generalized solutions, see [9, pp. 13-16, 163-172], [10, pp. 224229], and also [7].

Remark 2. Concerning the problems of uniqueness, regularity-or more generally, coherence, see $[2,3,10,12,1,5-7]$ of the generalized solutions whose existence is characterized in Theorem 1 , the situation at present is roughly as follows. Within the more particular framework of $[2,3]$, see also $[5,6,10$, $12,1]$, rather strong uniqueness, regularity and coherence results have been obtained for large classes of linear and nonlinear PDEs. On the other hand, in [7], the framework used is still more general than in this paper, in the sense that the mappings (2.18) are replaced by mappings

$$
T(D): E_{1} \rightarrow E_{2}
$$


where $E_{2}=\mathscr{S} / \mathscr{V} \in V S^{0}(\Omega)$ is a quotient vector space which need not be a quotient algebra.

Within this more general setting (4.70), and for rather general semilinear hyperbolic systems in two independent variables, existence and uniqueness is proved for the Cauchy problem with rough initial data. Rather surprisingly, precisely because of the more general nature of the framework in (4.70), particularly strong coherence results are proved for the unique generalized solutions of the mentioned type of Cauchy problems, results which are shown to be impossible within the framework of $[2,3]$, this being one of the outstanding features of [7].

However, the choice of the setting in $(2.18),(4.70)$ or that in $[2,3]$ may be influenced by considerations other than the strongest possible coherence of generalized solutions. Indeed, for the global version of the Cauchy-Kovalevskaia theorem in [12], the setting which proves to be useful is the following particular form of (2.18)

$$
T(D): A_{1} \rightarrow A_{2}
$$

where both $A_{1}$ and $A_{2}$ are quotient algebras of generalized functions.

Remark 3. The result in [12] which, within the setting of (4.71), proves the global existence of generalized solutions on the whole of the domain of analyticity of arbitrary nonlinear PDEs, can be seen as a particular case of the result in Theorem 1 above.

Indeed, the essence of the proof in [12] comes down to the fact that one can construct sequences of functions $s$ in (3.2)-(3.5) such that

$$
w_{s} \in \mathscr{I}_{\text {nd }}(\Omega)
$$

where $\mathscr{J}_{\text {nd }}(\Omega)$ is the set of all the sequences $w \in\left(\mathscr{C}^{0}(\Omega)\right)^{\mathbf{N}}$ which satisfy the condition

$$
\begin{aligned}
& \exists \Gamma \subset \Omega \text { closed, nowhere dense: } \\
& \forall x \in \Omega \backslash \Gamma: \\
& \exists \mu \in \mathbf{N}, V \subset \Omega \backslash \Gamma \text { neighborhood of } x: \\
& \forall \nu \in \mathbf{N}, \quad \nu \geq \mu, y \in V: \\
& \quad w_{\nu}(y)=0 .
\end{aligned}
$$

The important fact is that $\mathscr{I}_{\text {nd }}(\Omega)$ is an ideal in $\left(\mathscr{C}^{0}(\Omega)\right)^{\mathbf{N}}$, called the nowhere dense ideal, which obviously satisfies the neutrix condition (2.10). This makes it possible to easily secure the neutrix condition (4.57) in Theorem 1, and then set up the framework in (4.71).

\section{THE NEUTRIX CONDITION AS A DENSELY VANISHING CONDITION ON IDEALS}

The neutrix condition (2.10) first comes into the picture as the rather trivial necessary and sufficient condition for the algebra embedding in (2.12). Then, in (4.57), it nevertheless proves to give the characterization for the existence of generalized solutions of a large class of nonlinear PDEs.

This power of the neutrix condition (2.10) when applied to ideals $\mathscr{I}$ in subalgebras $\mathscr{A}$ of $\left(\mathscr{C}^{0}(\Omega)\right)^{\mathbf{N}}$ should not come as a surprise. Indeed, as seen in [9, pp. 75-88 and 10, pp. 306-315], the neutrix condition happens also to 
characterize the existence of a very large class of chains of differential algebras of generalized functions, chains which contain the L. Schwartz distributions, and incorporate as particular cases various distribution multiplications encountered in the literature, such as for instance that in [2, 3], for details on such multiplications see the references in $[9,10,11]$.

The form of the neutrix condition (2.10) has the significant advantage of being a particularly simple algebraic condition on the ideal $\mathscr{I}$, with the clear geometric meaning that $\mathscr{I}$ is off diagonal in $\left(\mathscr{C}^{0}(\Omega)\right)^{\mathbf{N}}$. However, except for that, the neutrix condition (2.10) does not give an explicit insight into the structure of the respective ideals $\mathscr{I}$. It is therefore of special interest to obtain alternative characterizations for the ideals $\mathscr{J}$ which satisfy the neutrix condition, characterizations which can give deeper insight into their structure. Indeed, as seen in (4.2) and (4.57), the structure of the ideals $\mathscr{I}$ which appear in quotient algebras $A=\mathscr{A} / \mathscr{I} \in A L(\Omega)$ can give a direct and explicit understanding of the conditions of solvability of nonlinear PDEs.

In this section, such an alternative characterization is presented, according to which, in a certain sense specified later, the sequences of functions $w \in \mathscr{J}$ have to vanish asymptotically on dense subsets of $\Omega$.

Let us now turn to the details. Our problem is the following. Given an inclusion diagram

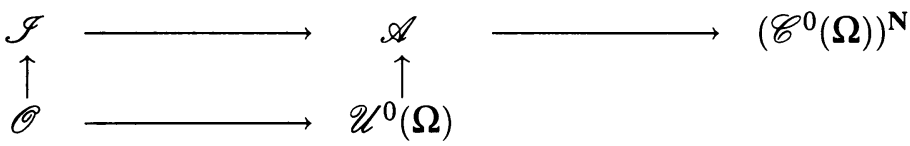

where $\mathscr{A}$ is a subalgebra and $\mathscr{I}$ is an ideal in $\mathscr{A}$ which satisfies the neutrix condition

$$
\mathscr{I} \cap \mathscr{U}^{0}(\Omega)=\mathscr{O}
$$

we want to find equivalent conditions on $\mathscr{I}$, which give a better explicit understanding of the structure of $\mathscr{I}$.

It should be noted from the beginning that this problem of structural characterizations of $\mathscr{I}$ seems to be nontrivial. Indeed, it can often happen that when constructing generalized solutions for lilnear or nonlinear PDEs, we shall encounter in (5.1) the situation where

$$
\mathscr{A} \varsubsetneqq\left(\mathscr{C}^{0}(\Omega)\right)^{\mathbf{N}}
$$

and

$$
\mathscr{I} \text { is not an ideal in }\left(\mathscr{C}^{0}(\Omega)\right)^{\mathbf{N}} \text {. }
$$

Since obviously

$$
\left(\mathscr{C}^{0}(\Omega)\right)^{\mathbf{N}}=\mathscr{C}(\mathbf{N} \times \Omega)
$$

the situation in (5.3), (5.4) means that we are dealing with the structure of ideals $\mathscr{I}$ in strict subalgebras $\mathscr{A}$ of a ring of continuous functions, namely $\mathscr{C}(\mathbf{N} \times \Omega)$, a problem well known for its difficulty, see [15].

Finally, we should note that from the point of view of the structure of $\mathscr{I}$, the problem in (5.1), (5.2) has the following equivalent and simpler formulation, in which the subalgebra $\mathscr{A}$ does no longer appear: find the structure of subalgebras

$$
\mathscr{I} \subset\left(\mathscr{C}^{0}(\Omega)\right)^{\mathbf{N}}
$$


which satisfy the conditions

$$
\mathscr{I} \cdot \mathscr{U}^{0}(\Omega) \subset \mathscr{I}
$$

and

$$
\mathscr{I} \cap \mathscr{U}^{0}(\Omega)=\mathscr{O} \text {. }
$$

Obviously, the neutrix condition (5.8) will imply that $\mathscr{I}$ is a strict subalgebra in $\left(\mathscr{C}^{0}(\Omega)\right)^{\mathbf{N}}$.

And now, we can turn to the "dense vanishing" characterization of subalgebras $\mathscr{J}$ in $(5.6)-(5.8)$.

The conjecture about it has emerged earlier, following the study of the essence of various sufficient conditions for obtaining (5.6)-(5.8), see for details [8, pp. 39-59, 132-138, 9, pp. 173-198]. Indeed, for $w \in\left(\mathscr{C}^{0}(\Omega)\right)^{\mathbf{N}}$, let us define its vanishing set by

$$
\Omega_{w}=\left\{x \in \Omega\left|\inf _{\nu \in \mathbf{N}}\right| w_{\nu}(x) \mid=0\right\} .
$$

Then, the following sufficient condition for (5.8) results easily.

Proposition 5. Given any subset $\mathscr{I} \subset\left(\mathscr{C}^{0}(\Omega)\right)^{\mathbf{N}}$, if

$$
\forall w \in \mathscr{J}: \Omega_{w} \text { is dense in } \Omega
$$

then

$$
\mathscr{I} \cap \mathscr{U}^{0}(\Omega) \subset \mathscr{O} \text {. }
$$

Proof. Assume that for certain $w \in \mathscr{I}$ and $\psi \in \mathscr{C}^{0}(\Omega)$ we have

$$
w=u(\psi)
$$

then

$$
\psi(x)=w_{\nu}(x), \quad \forall \nu \in \mathbf{N}, x \in \Omega
$$

hence (5.9) implies that

$$
|\psi(x)|=\inf _{\nu \in \mathbf{N}}\left|w_{\nu}(x)\right|=0, \quad \forall x \in \Omega_{x} .
$$

Now (5.12) and (5.10) as well as the continuity of $\psi$ on $\Omega$ will yield

$$
\psi(x)=0, \quad \forall x \in \Omega \text {. }
$$

It is easy to see that $\mathscr{J}_{\text {nd }}(\Omega)$ defined in (4.73), does satisfy the "densely vanishing" condition (5.10).

As the main result of this section, in Theorem 2 it will be shown that the "dense vanishing" condition (5.10) is equivalent to the neutrix condition, within a rather large and natural class of inclusion diagrams (5.1). The basic property used for that purpose is presented now.

Proposition 6. If $w \in\left(\mathscr{C}^{0}(\Omega)\right)^{\mathbf{N}}$ and $\Omega_{w}$ is not dense in $\Omega$, then

$$
\begin{aligned}
& \exists \Omega^{\prime} \subset \Omega \text { nonvoid, open, } c>0: \\
& \forall \nu \in \mathbf{N}, \quad x \in \Omega^{\prime}:
\end{aligned}
$$

$$
\left|w_{\nu}(x)\right| \geq c .
$$


Proof. As $\Omega_{w}$ is not dense in $\Omega$, it follows that

$$
\begin{aligned}
& \exists x \in \Omega, \quad \varepsilon>0: \\
& B(x, \varepsilon) \cap \Omega_{w}=\varnothing .
\end{aligned}
$$

Define now $\delta: \Omega \rightarrow[0, \infty)$ by

$$
\delta(y)=\inf _{\nu \in \mathbf{N}}\left|w_{\nu}(y)\right|, \quad \forall y \in \Omega .
$$

Obviously, $\delta$ is upper semicontinuous. Therefore, the set $D \subset \Omega$ of discontinuities of $\delta$ is of first Baire category in $\Omega$. In this way it follows that $B(x, \epsilon) \backslash D \neq \varnothing$ thus we can find $y \in B(x, \epsilon)$ such that $\delta$ is continuous at $y$. But then, in view of (5.14), we have $\delta(y)>0$ hence (5.13) follows by taking (5.15) into account.

At this stage, we shall restrict the class of inclusion diagrams (5.1), (5.2) by noting that many of the spaces of generalized functions have a sheaf structure, see [10, pp. 131-133]. For instance, in the case of the L. Schwartz distributions, we have a natural mapping

$$
\Omega \supset \Omega^{\prime} \text { open } \mapsto \mathscr{D}^{\prime}(\Omega)
$$

which turns $\mathscr{D}^{\prime}(\Omega)$ into a sheaf of sections over $\Omega$, see [13].

In view of the above, a subalgebra $\mathscr{I}$ in $\left(\mathscr{C}^{0}(\Omega)\right)^{\mathbf{N}}$, see (5.6), will be called local, if and only if

$$
\forall \boldsymbol{\Omega}^{\prime} \subset \boldsymbol{\Omega} \text { nonvoid, open: }\left.\mathscr{I}\right|_{\boldsymbol{\Omega}^{\prime}} \cap \mathscr{U}^{0}\left(\boldsymbol{\Omega}^{\prime}\right)=\mathscr{O} \text {. }
$$

Here we recall that we have

$$
\left.\mathscr{I}\right|_{\Omega^{\prime}}=\left\{\left.w\right|_{\Omega^{\prime}} \mid w \in \mathscr{I}\right\}
$$

where, for $w=\left(w_{0}, \ldots, w_{\nu}, \ldots\right) \in\left(\mathscr{C}^{0}(\Omega)\right)^{\mathbf{N}}$, we denoted

$$
\left.w\right|_{\Omega^{\prime}}=\left(\left.w_{0}\right|_{\Omega^{\prime}}, \ldots,\left.w_{\nu}\right|_{\Omega^{\prime}}, \ldots\right)
$$

with

$$
\left.\mathscr{C}^{0}(\Omega) \ni h \mapsto h\right|_{\Omega^{\prime}} \in \mathscr{C}^{0}\left(\mathbf{\Omega}^{\prime}\right)
$$

being the usual restriction of the function $h$ to $\Omega^{\prime}$. It follows easily that $\mathscr{I}_{\text {nd }}(\Omega)$ is local.

Finally, a subalgebra $\mathscr{A}$ in $\left(\mathscr{C}^{0}(\Omega)\right)^{\mathbf{N}}$ is called full, if and only if

$$
\begin{aligned}
& \forall \Omega^{\prime} \subset \Omega \text { nonvoid, open, } t \in \mathscr{A}: \\
& \left.\quad\left(\begin{array}{c}
\exists c>0: \\
\forall \nu \in \mathbf{N}, x \in \Omega^{\prime}: \\
\left|t_{\nu}(x)\right| \geq c
\end{array}\right) \Rightarrow \frac{1}{\left.t\right|_{\Omega^{\prime}}} \in \mathscr{A}\right|_{\Omega^{\prime}}
\end{aligned}
$$

Obviously, $\mathscr{A}=\left(\mathscr{C}^{0}(\Omega)\right)^{\mathbf{N}}$ is full. Therefore $\mathscr{I}_{\mathrm{n}}(\Omega)$ is a local ideal in the full algebra $\left(\mathscr{C}^{0}(\Omega)\right)^{\mathbf{N}}$.

We also note that conditions (5.17) and (5.18), obviously recall (4.12), (4.16) and the definition of a nontrivial $T(D)$ in $\S 4$, in all of which an arbitrary nonvoid, open $\Omega^{\prime} \subset \Omega$ is present.

And now, the main result in this section. 
Theorem 2. Given $\mathscr{A}$ a full subalgebra in $\left(\mathscr{C}^{0}(\Omega)\right)^{\mathbf{N}}$ and $\mathscr{I}$ a local ideal in $\mathscr{A}$. Then, the neutrix condition

$$
\mathscr{I} \cap \mathscr{U}^{0}(\Omega)=\mathscr{O}
$$

and the "densely vanishing" condition

$$
\forall w \in \mathcal{I}: \Omega_{w} \text { is dense in } \Omega
$$

are equivalent.

Proof. The implication (5.20) $\Rightarrow(5.19)$ follows from Proposition 5. Conversely, assume that $\Omega_{w}$ is not dense in $\Omega$, for some $w \in \mathscr{I}$. Then we have (5.13). But obviously,

$$
\left.\left.\left.w\right|_{\Omega^{\prime}} \in \mathscr{I}\right|_{\Omega^{\prime}} \subset \mathscr{A}\right|_{\Omega^{\prime}}
$$

therefore (5.18) implies that $1 /\left.\left.w\right|_{\Omega^{\prime}} \in \mathscr{A}\right|_{\Omega^{\prime}}$. It follows that

$$
u(1)=\left.\left.\left.\left(\left.w\right|_{\Omega^{\prime}}\right) \cdot\left(\frac{1}{\left.w\right|_{\Omega^{\prime}}}\right) \in \mathscr{I}\right|_{\Omega^{\prime}} \cdot A\right|_{\Omega^{\prime}} \subset \mathscr{I}\right|_{\Omega^{\prime}}
$$

since $\left.\mathscr{I}\right|_{\Omega^{\prime}}$ is an ideal in $\left.\mathscr{A}\right|_{\Omega^{\prime}}$. But (5.21) contradicts (5.17).

The "neutrix calculus", in particular, the neutrix condition (2.10), has first been introduced in [14], in connection with an abstract model for the study of large classes of asymptotic expansions. The setting in [14] is in essence the following. Given an Abelian group $G$ and an arbitrary infinite set $X$, a subgroup $\mathscr{N} \subset G^{X}$ is called a neutrix, if and only if

$$
\begin{aligned}
& \forall f \in \mathscr{N}, \quad \gamma \in G: \\
& \quad\left(\begin{array}{l}
\forall x \in X: \\
f(x)=\gamma
\end{array}\right) \Rightarrow \gamma=0
\end{aligned}
$$

in which case the functions $f \in \mathscr{N}$ will be called $\mathscr{N}$-negligible, see [9, pp. 285-288, 10, pp. 267-270].

However, the power of the neutrix condition (2.10) comes into play in a significant manner within the more particular framework of $(2.7)$, when it is applied to ideals $\mathscr{I}$, see [9, pp. 75-88, 10, pp. 306-315].

\section{Dense Vanishing IN THE CASE OF SMOOTh IDEALS}

In a rather surprising manner, it happens that the "densely vanishing" property (5.20) can be significantly strengthened in the case of subalgebras in $(\mathscr{C} \infty(\Omega))^{\mathbf{N}}$. Indeed, as in Theorem 2, suppose given a full subalgebra $\mathscr{A}$ in $\left(\mathscr{C}^{0}(\Omega)\right)^{\mathbf{N}}$ and a local ideal $\mathscr{I}$ in $\mathscr{A}$. Let us define

$$
\mathscr{I}^{\infty}=\left\{w \in \mathscr{I} \cap\left(\mathscr{C}^{\infty}(\boldsymbol{\Omega})\right)^{\mathbf{N}} \mid \forall p \in \mathbf{N}^{n}: D^{p} w \in \mathscr{I}\right\}
$$

which in view of the Leibnitz rule of product derivative, will be an ideal in

$$
\mathscr{A}^{\infty}=\left\{t \in \mathscr{A} \cap\left(\mathscr{C}^{\infty}(\Omega)\right)^{\mathbf{N}} \mid \forall p \in \mathbf{N}^{n}: D^{p} t \in \mathscr{A}\right\} .
$$

It follows easily that, as an example, we have

$$
\left(\mathscr{I}_{\text {nd }}(\Omega)\right)^{\infty}=\mathscr{I}_{\text {nd }}(\Omega) \cap\left(\mathscr{C}^{\infty}(\Omega)\right)^{\mathbf{N}}, \quad\left(\left(\mathscr{C}^{0}(\Omega)\right)^{\mathbf{N}}\right)^{\infty}=\left(\mathscr{C}^{0}(\Omega)\right)^{\mathbf{N}} .
$$

As seen in [8-12] the ideal $\left(\mathscr{I}_{\text {nd }}(\Omega)\right)^{\infty}$ plays a crucial role in the construction of generalized solutions for wide classes of nonlinear PDEs. 
Now, for $w \in \mathscr{J}^{\infty}$ let us denote

$$
\Omega(w)=\left\{x \in \Omega\left|\forall p \in \mathbf{N}^{n}: \inf _{\nu \in \mathbf{N}}\right| D^{p} w_{\nu}(x) \mid=0\right\}=\bigcap_{p \in \mathbf{N}^{n}} \Omega_{D^{p} w} .
$$

Theorem 3. The ideal $\mathscr{I} \infty$ satisfies the following "densely vanishing" condition

$$
\forall w \in \mathscr{J}^{\infty}:
$$

$$
\Omega(w) \text { is dense in } \Omega \text {. }
$$

Proof. Assume (6.4) is false and take $w \in \mathscr{I}^{\infty}, x \in \Omega$ and $\epsilon>0$ such that

$$
B(x, \epsilon) \cap \Omega(w)=\varnothing .
$$

For $p \in \mathbf{N}^{n}$ we define $\delta_{p}: \Omega \rightarrow[0, \infty)$ by

$$
\delta_{p}(y)=\inf _{\nu \in \mathbf{N}}\left|D^{p} w_{\nu}(y)\right|, \quad \forall y \in \Omega .
$$

Then $\delta_{p}$ is upper semicontinuous. Therefore the set $D_{p} \subset \Omega$ of discontinuities of $\delta_{p}$ is of first Baire category in $\Omega$. In this way

$$
D=\bigcup_{p \in \mathbf{N}^{n}} D^{p} \text { is of first Baire category in } \Omega .
$$

It follows that we can take $y \in B(x, \epsilon) \backslash D$ in which case (6.5) yields $\exists p \in$ $\mathbf{N}^{n}: y \notin \Omega_{D_{w}^{p}}$ and in view of (6.6), we obtain $\delta_{p}(y)>0$ thus (5.13) will hold for $t=D^{p} w$. But $w \in \mathscr{I}^{\infty}$ and (6.1) imply that $t=D^{p} w \in \mathscr{I} \subset \mathscr{A}$ and we can apply (5.18), obtaining the relation

$$
\left.\frac{1}{\left.t\right|_{\Omega^{\prime}}} \in \mathscr{A}\right|_{\Omega^{\prime}}
$$

Then, as in (5.21), it follows that

$$
\left.u(1) \in \mathscr{I}\right|_{\Omega^{\prime}}
$$

and (5.17) is contradicted.

The "densely vanishing" condition (6.4) can further be strengthened. A subalgebra $\mathscr{I}$ in $\left(\mathscr{C}^{0}(\Omega)\right)^{\mathbf{N}}$ is called circled, if and only if

$$
\forall w \in \mathscr{I}:|w| \in \mathscr{J} .
$$

Obviously, $\mathscr{I}_{\text {nd }}(\Omega)$ is circled.

Given a vector subspace $\mathscr{W} \subset \mathscr{J}^{\infty}$ let us denote

$$
\Omega(\mathscr{W})=\bigcap_{w \in \mathscr{W}} \Omega(w)
$$

Theorem 4. Under the conditions in Theorem 3, suppose that $\mathscr{I}$ is circled. Then the ideal $\mathscr{I}^{\infty}$ satisfies the following "densely vanishing" condition

$$
\begin{aligned}
& \forall \mathscr{W} \subset \mathscr{I}^{\infty} \text { countably infinite-dimensional vector subspace: } \\
& \Omega(\mathscr{W}) \text { is dense in } \Omega \text {. }
\end{aligned}
$$

Proof. Assume that (6.9) is false for a certain countably infinite-dimensional vector subspace $\mathscr{W} \subset \mathscr{J}^{\infty}$ generated by a Hamel basis

$$
w^{0}, \ldots, w^{m}, \cdots \in \mathscr{W} \text {. }
$$


Let us take $x \in \Omega$ and $\varepsilon>0$ such that

$$
B(x, \varepsilon) \cap \Omega(\mathscr{W})=\varnothing .
$$

For $m \in \mathbf{N}$ and $p \in \mathbf{N}^{n}$ we define

$$
w^{m, p}=\left|D^{p} w^{0}\right|+\cdots+\left|D^{p} w^{m}\right| \in \mathscr{I}
$$

and $\delta_{m, p}: \Omega \rightarrow[0, \infty)$ by

$$
\delta_{m, p}(y)=\inf _{\nu \in \mathbf{N}}\left|\left(w^{m, p}\right)_{\nu}(y)\right|, \quad \forall y \in \Omega .
$$

In this way $\delta_{m, p}$ are upper semicontinuous. Therefore, denoting by $D_{m, p} \subset \Omega$ the set of discontinuities of $\delta_{m, p}$, it follows that $D_{m, p}$ is of first Baire category in $\Omega$.

In this way

$$
D=\bigcup_{m \in \mathbf{N}} \bigcup_{p \in \mathbb{N}^{n}} D_{m, p} \text { is of first Baire category in } \Omega
$$

and we can take

$$
y \in B(x, \epsilon) \backslash D .
$$

Then (6.11) implies that

$$
\exists w \in \mathscr{W}, p \in \mathbf{N}^{n}: y \notin \Omega_{D^{p} w} .
$$

But according to $(6.10)$, we obtain

$$
w=\lambda_{0} w^{0}+\cdots+\lambda_{m} w^{m}
$$

for suitable $\lambda_{0}, \ldots, \lambda_{m}$. Therefore, in view of (6.12), (6.16) and Lemma 2 below, we have $\Omega_{w^{m, p}} \subset \Omega_{D^{p} w}$ hence (6.15) implies that $y \notin \Omega_{w^{m, p}}$ and then (6.13) will give $\delta_{m, p}(y)>0$.

It follows that (5.13) holds for $t=w^{m, p}$. But (6.12) implies that $t=w^{m, p} \in$ $\mathscr{I} \subset \mathscr{A}$. Thus in view of (5.18) we obtain

$$
\left.\frac{1}{\left.t\right|_{\Omega^{\prime}}} \in \mathscr{A}\right|_{\Omega^{\prime}}
$$

and similar to (5.21), the relation results $u(1) \in \mathscr{I}_{\Omega_{\Omega^{\prime}}}$ which contradicts (5.17).

Lemma 2. If $w \in\left(\mathscr{C}^{0}(\Omega)\right)^{\mathbf{N}}$ then

$$
\Omega_{|w|}=\Omega_{w} .
$$

More generally, if $w^{0}, \ldots, w^{m} \in\left(\mathscr{C}^{0}(\Omega)\right)^{\mathbf{N}}$ and $\lambda_{0}, \ldots, \lambda_{m} \in \mathbf{R}$ then

$$
\Omega_{\left|w^{0}\right|+\cdots+\mid w^{m \mid}} \subset \Omega_{\lambda_{0} w^{0}+\cdots+\lambda_{m} w^{m}} .
$$

Proof. In view of (5.9), the relation (6.17) is obvious. Take now $\nu \in \mathbf{N}$ and $x \in \Omega$, then

$$
\begin{aligned}
& \left|\lambda_{0}\left(w^{0}\right)_{\nu}(x)+\cdots+\lambda_{m}\left(w^{m}\right)_{\nu}(x)\right| \\
& \quad \leq\left|\lambda_{0}\right| \cdot\left|\left(w^{0}\right)_{\nu}(x)\right|+\cdots+\left|\lambda_{m}\right| \cdot\left|\left(w^{m}\right)_{\nu}(x)\right| \\
& \quad \leq|\lambda| \cdot\left(\left|\left(w^{0}\right)_{\nu}(x)\right|+\cdots+\left|\left(w^{m}\right)_{\nu}(x)\right|\right)
\end{aligned}
$$

for every $\lambda \in \mathbf{R}$, such that $\max \left\{\left|\lambda_{0}\right|, \ldots,\left|\lambda_{m}\right|\right\} \leq|\lambda|$.

But (5.9), (6.17) and (6.19) obviously imply (6.18). 


\section{THE CASE OF NORMAL IDEALS}

The increasingly stronger "densely vanishing" conditions (5.20), (6.4) and (6.9) seem to point to a deeper property involved, whose full explicitation is still an open problem. This is illustrated for instance by the fact that the above "densely vanishing" conditions (5.20), (6.4) and (6.9) can be obtained under the following alternative assumptions, when $\mathscr{I}$ is a subalgebra in $\left(\mathscr{C}^{0}(\Omega)\right)^{\mathbf{N}}$ which satisfies the neutrix condition

$$
\mathscr{J} \cap \mathscr{U}^{0}(\Omega)=\mathscr{O}
$$

and in addition, it is also normal, [4], that is, it has the property

$$
\begin{aligned}
& \forall w \in\left(\mathscr{C}^{0}(\Omega)\right)^{\mathbf{N}}: \\
& \quad\left(\begin{array}{c}
\exists z \in \mathscr{I}: \\
\forall \nu \in \mathbf{N}, x \in \Omega: \\
\left|w_{\nu}(x)\right| \leq\left|z_{\nu}(x)\right|
\end{array}\right) \Rightarrow w \in \mathscr{I} .
\end{aligned}
$$

Indeed, the proofs of Theorems 2-4 will go through with the following modification. When obtaining (5.13) in the respective proofs, we no longer use (5.18). Instead we note that we can use the property

$$
\begin{aligned}
& \exists a \in \mathscr{C}^{0}(\Omega) \\
& \quad(*) \quad a \neq 0 \\
& (* *) \quad \forall \nu \in \mathbf{N}, \quad x \in \Omega: 0 \leq a(x) \leq\left|w_{\nu}(x)\right|
\end{aligned}
$$

which in view of (7.2) will imply $u(a) \in \mathscr{I} \cap \mathscr{U}^{0}(\Omega)$. Then owing to (*) in (7.3), the neutrix condition (7.1) is contradicted. We note as an example that $\mathscr{I}_{\text {nd }}(\Omega)$ is obviously normal.

\section{ACKNOWLEDGEMENT}

The author is most grateful to M. Oberguggenberger for his particularly useful contribution in correcting certain errors and suggesting several improvements in an earlier version of the paper.

\section{REFERENCES}

1. H. A. Biagioni, Introduction to a nonlinear theory of generalized functions, Notas de Matematica, IMECC, Campinas, Brazil, 1988.

2. J. F. Colombeau, New generalized functions and multiplication of distributions, NorthHolland, Amsterdam, 1984.

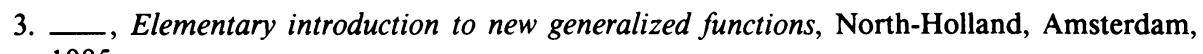
1985.

4. G. Köthe, Topological vector spaces. I, Springer, Heidelberg, 1960.

5. M. Oberguggenberger, Weak limits of solutions to semilinear hyperbolic systems, Math. Ann. 274 (1986), 599-607.

6. __ Generalized solutions to semilinear hyperbolic systems, Mh. Math. 103 (1987), 133144.

7. __ Semilinear wave equations with rough initial data: generalized solutions, P. Antosik and A. Kaminski (Eds.), Generalized Functions and Convergence, World Scientific Publ., London, 1990.

8. E. E. Rosinger, Distributions and nonlinear partial differential equations, Springer, Berlin, 1978. 
9. __ Nonlinear partial differential equations, sequential and weak solutions, North-Holland, Amsterdam, 1980.

10. E. E. Rosinger, Generalized solutions of nonlinear partial differential equations, NorthHolland, Amsterdam, 1987.

11. Nonlinear partial differential equaations, an algebraic view of generalized solutions, North-Holland, Amsterdam, 1990.

12. __ Global version of the Cauchy-Kovalevskaia theorem for nonlinear PDEs, Acta Appl. Math. (to appear).

13. J. A. Seebach, Jr., L. A. Seebach, and L. A. Steen, What is a sheaf?, Amer. Math. Monthly (1970), 681-703.

14. J. C. Van der Corput, Introduction to neutrix calculus, J. Analyse Math. 7 (1959), 281-398.

15. R. C. Walker, The Stone-Čech compactification, Springer, Berlin, 1974.

Department of Mathematics, University of Pretoria, Pretoria 0002, South Africa 